\title{
A Severe Abdominal Injury Caused by Concertina Razor Wire
}

\author{
Jung Hee Choi', Seung Je $\mathrm{Go}^{2}$, Young Hoon Sul', Jin Bong Ye', Jin Young Lee', \\ Dong Hee Ryư ${ }^{3}$, Mou Seop Lee ${ }^{4}$ \\ ${ }^{1}$ Department of Anesthesiology and Pain Medicine, ${ }^{2}$ Department of Trauma Surgery, ${ }^{3}$ Department of Surgery, ${ }^{4}$ Department of \\ Neurosurgery Chungbuk National University Hospital, Cheongju, Korea
}

We report a case of a severe abdominal injury caused by concertina razor wire used in military units. A 15-year-old man had herniated his small bowel and colon through a large abdominal open wound caused by the concertina razor wire. Three abdominal operations were performed including damage control surgery due to the severe intra-peritoneal contamination and abdominal wall infection.

(Trauma Image Proced 2017(2):75-78)

Key Words: Concertina razor wire; Damage control surgery; Intra-peritoneal contamination

\section{CASE}

A 15-year-old man presented to the emergency department with a severe abdominal injury caused by falling over concertina razor wire while riding a bicycle. The patient was alert, and his vital signs were within normal ranges with the exception of tachycardia. He had a herniated small intestine and colon through a large abdominal open wound (about $25 \mathrm{~cm}$ in length) caused by the concertina razor wire. Also, a part of the concertina razor wire was embedded in the abdominal cavity (Fig. 1). Bleeding through an open abdominal wound was not observed. An emergency operation was planned following a computed tomography (CT) scan of the abdominal region (Fig. 2, 3).

During the emergent laparotomy, we noticed observed no other organ injuries other than the section of intestine which was injured by a part of the concertina razor wire (still embedded in the abdominal cavity). The embedded razor wire was removed carefully (Fig. 4), and about $150 \mathrm{~cm}$ of the injured jejunum accompanied by severe mesenteric lacerations and multiple perforations was also resected. Then, a left hemicolectomy was performed due to a descending colon injury. A temporary closure of the abdominal region was performed after a thorough irrigation with saline irrigation was completed due to the severe intra-peritoneal contamination from bowel contents, leaves, dirt, and a piece of clothes which were found in the peritoneal cavity.

After two days, end-to-end anastomosis of the resected jejunum and end-colostomy at the left upper quadrant were performed with saline irrigation in the peritoneal cavity. Then, a closure of the abdominal wall with fascial a defect of about $9 \times 8 \mathrm{~cm}$ was performed. However, a wound infection with fasciitis around the

Received: November 5, 2017 Revised: November 10, 2017 Accepted: November 13, 2017

Correspondence to: Seung Je Go, Department of Trauma Surgery, Chungbuk National University Hospital, 776 1Sunhwan-ro, Seowon-gu, Cheongju-si, Chungcheongbuk-do, 28644, Korea

Tel: 82-43-269-7850, Fax: 82-43-269-8809, E-mail: rhtmdwp@hanmail.net

Copyright (c) 2017 Korean Association for Research, Procedures and Education on Trauma. All rights reserved.

@) This is an open-access article distributed under the terms of the Creative Commons Attribution Non-Commercial License (http://creativecommons.org/ licenses/by-nc/4.0) which permits unrestricted noncommercial use, distribution, and reproduction in any medium, provided the original work is properly cited 

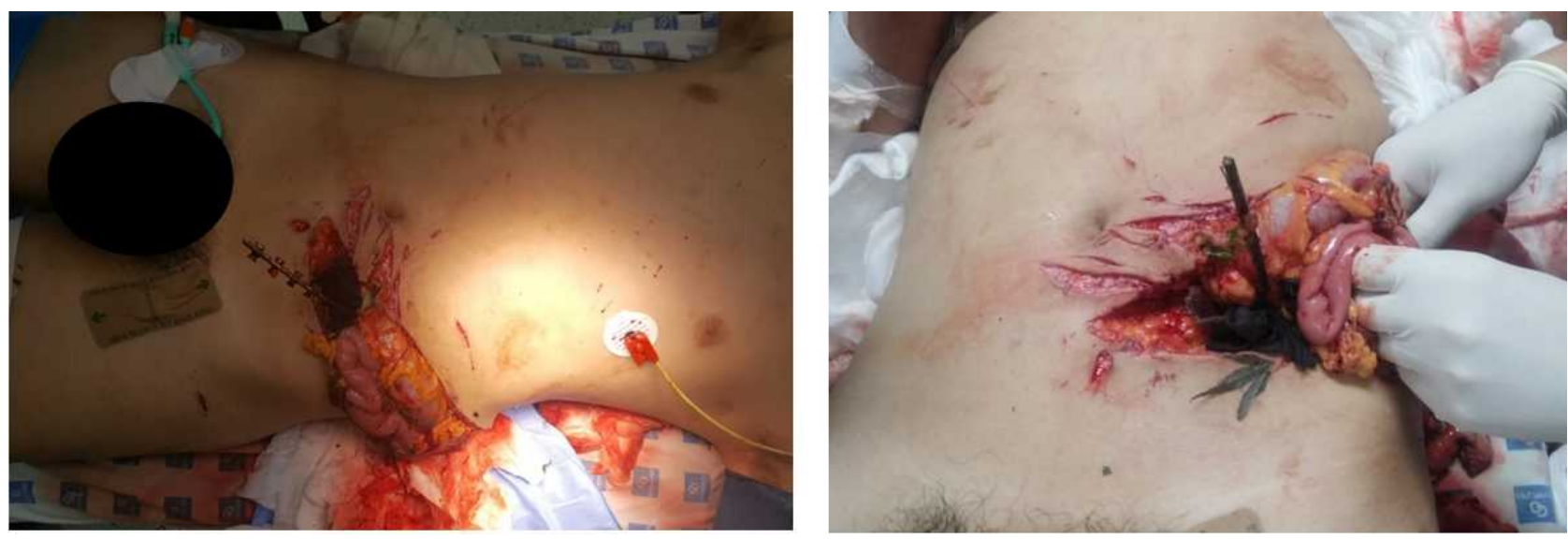

Fig. 1. Herniated small bowel and colon through the large abdominal open wound and a part of concertina razor wires were embedded in the abdominal cavity.
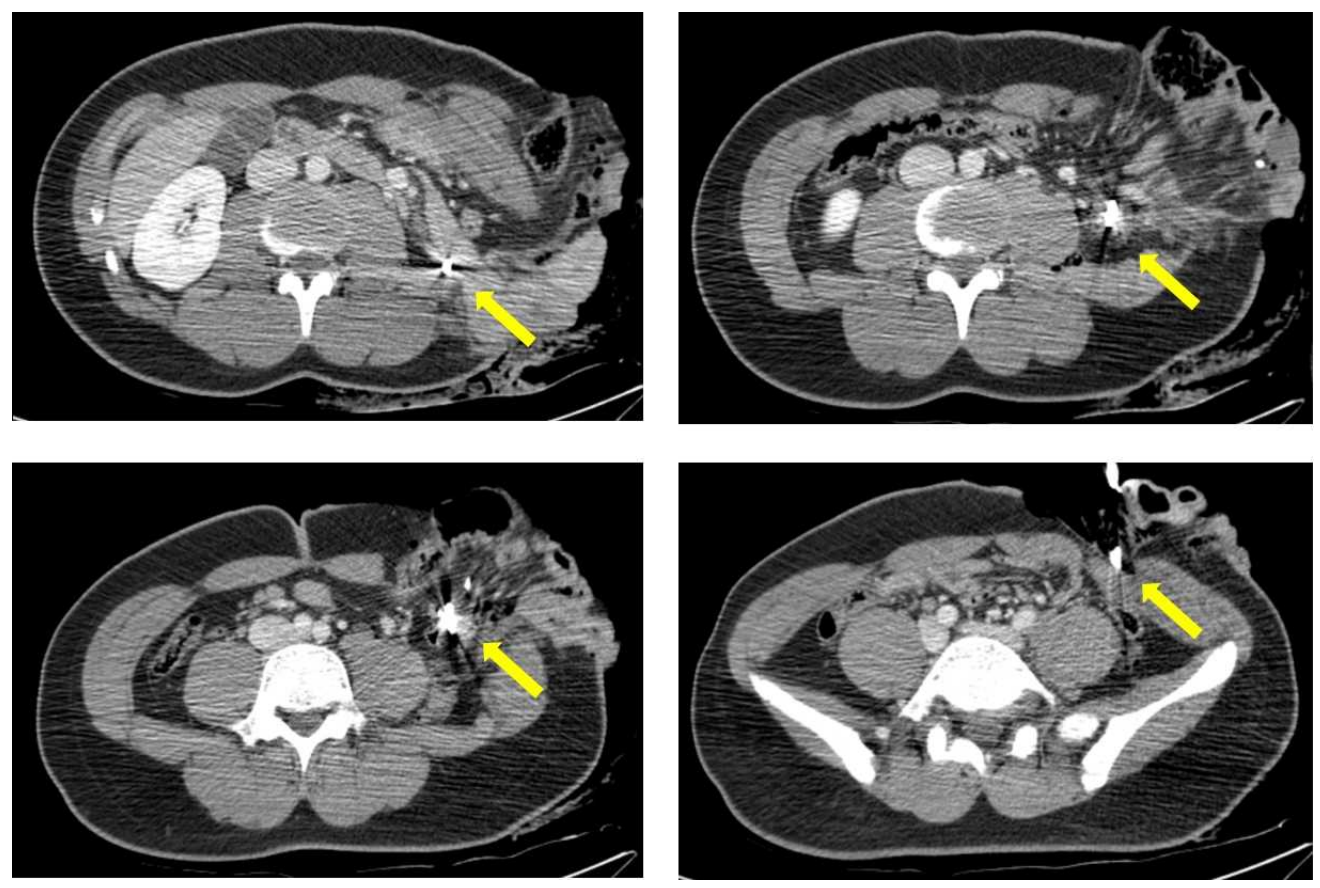

Fig. 2. Abdominal CT scan (axial view) demonstrates a herniated small bowel and descending colon through an open abdominal wound, and a foreign body (yellow arrow) was embedded in the abdominal cavity.

injury developed and gradually extended to the colostomy site. After the colostomy repositioning at the upper midline wound was performed (Fig. 5), the patient recovered and was discharged without any further more complications. We performed a reversal of the colostomy and a definitive closure of the fascial defect with a porcine dermal collagen after three months.

\section{Discussion}

The barbed wire is strong with sharp points all around it and is used to prevent people or animals from entering or leaving a place. Injuries caused by it usually involve piercing or scratching of the skin. But, the concertina razor wire, a variation of barbed wire that is 

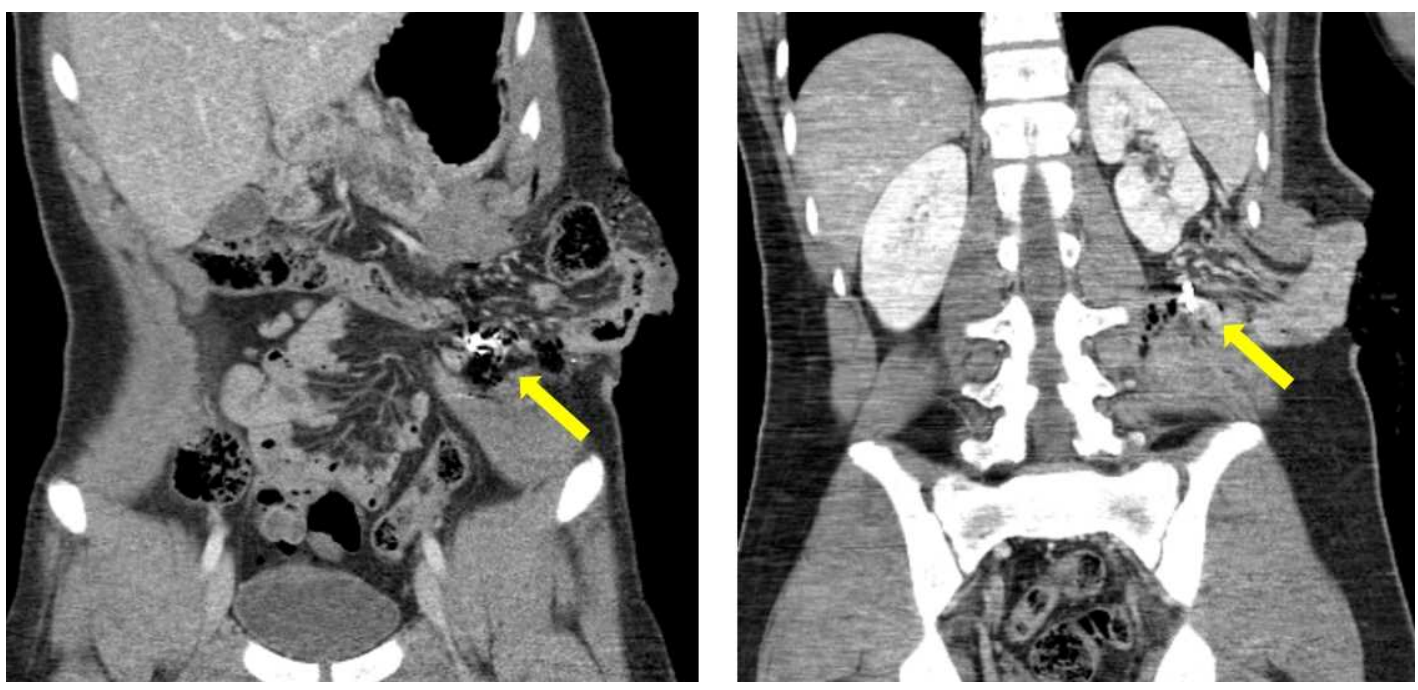

Fig. 3. Abdominal CT scan (coronal view) demonstrates a herniated small bowel and descending colon through the open abdominal wound, and the foreign body (yellow arrow) was embedded in the abdominal cavity.
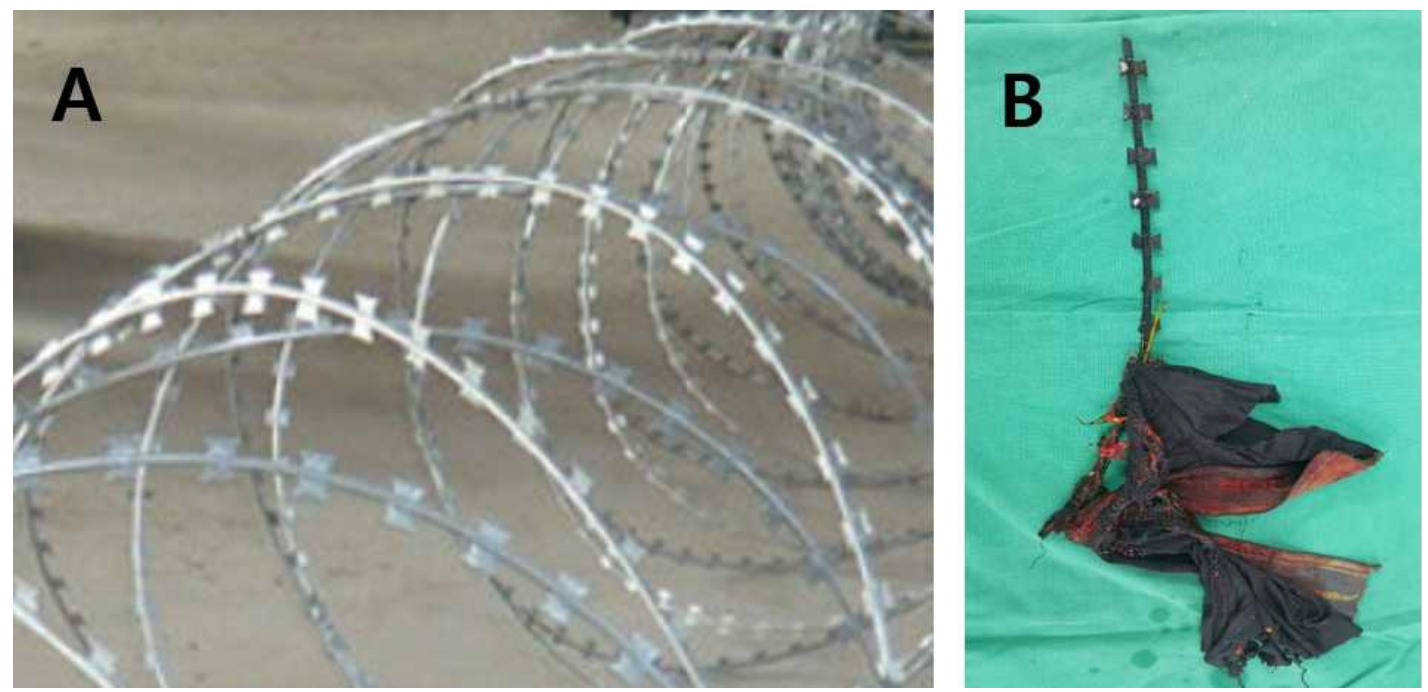

Fig. 4. (A) Concertina razor wire (B) A part of concertina razor wire with a piece of clothes embedded in the abdominal cavity.

used for military purposes, can cause deeper injuries, as was the case herein. Furthermore, if the concertina razor wire has kinetic energy as in our case, it can certainly cause fatalities.

A major concern in the management of colonic injuries is to determine whether a fecal diversion is necessary. The 2003 Cochrane review found no difference in mortality rates, abdominal infection or wound complications and concluded that the primary repair of penetrating colonic injury is as safe as diversion surgery (1).
However, patients with diabetes, cirrhosis or human immunodeficiency virus infection, transfusion of more than 6 units of packed red blood cells within 24 hours, destructive colonic injuries, heavy fecal contamination or a delay of more than 6 hours from injury to surgery are considered to be at high-risk of complications $(2,3,4)$. Therefore, in these cases, the injured bowel is resected, and ends are stapled without anastomosis. The abdomen may be left open with a temporary abdominal closure. A "second-look" laparotomy is usually conducted after 24 

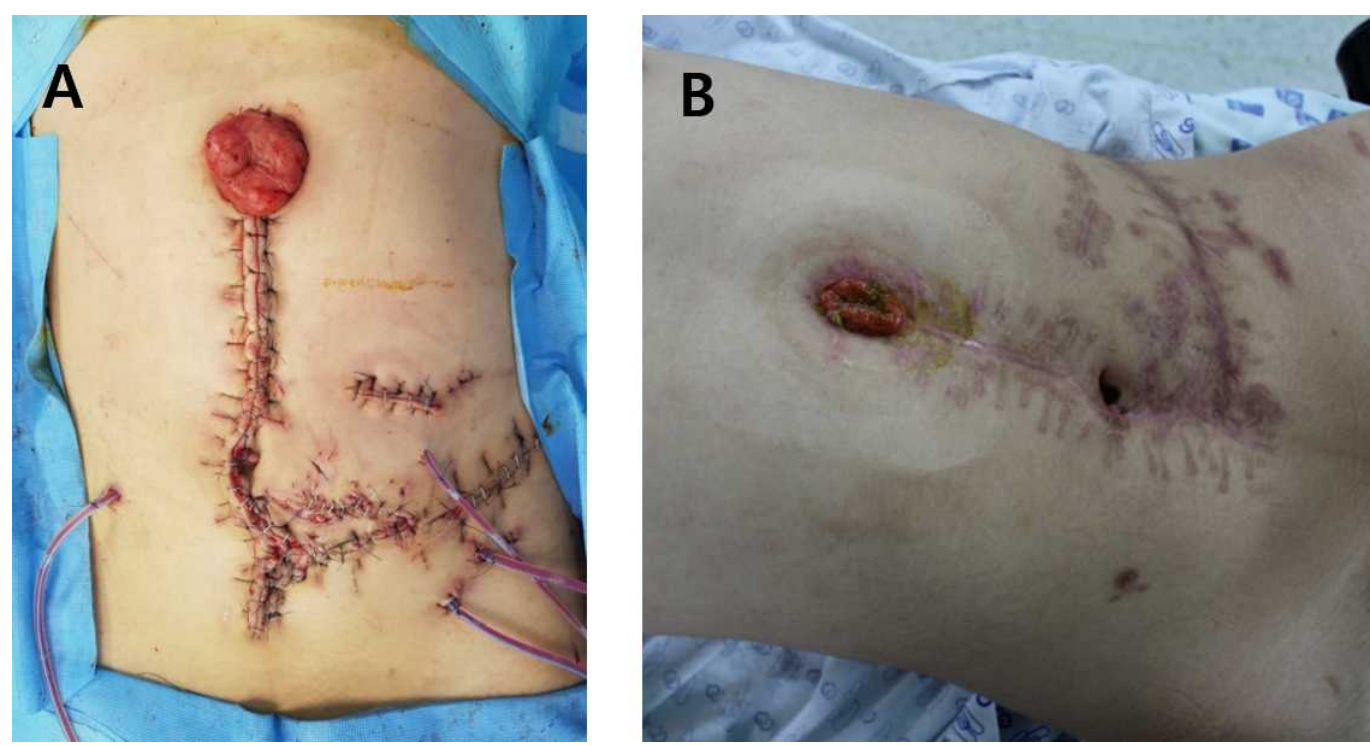

Fig. 5. (A) A colostomy repositioning at the upper midline wound was performed. (B) Recovery after colostomy repositioning.

- 48 hours once the patient's physiology normalizes. The colon can then be anastomosed at this time, or a stoma can be created (5).

We performed damage control surgery in our case because of the destructive colonic injuries and heavy fecal contamination and created a stoma of colostomy during the "second-look" operation. Given the observations reported herein, the damage control surgery should be considered as an effective approach not only in the case of massive bleeding but also to abate severe intra-peritoneal contamination.

\section{Conflict of Interest Statement}

No potential conflict of interest relevant to this article was reported.

\section{REFERENCES}

1. Nelson RL, Singer M. Direct (primary) repair for penetrating colon injuries. Cochrane Database Syst Rev 2003.

2. Stewart RM, Fabian TC, Croce MA, Pritchard FE, Minard G, Kudsk KA . Is resection with primary anastomosis following destructive colon wounds always safe? Am J Surg 1994;168: $316-9$.

3. Sharpe JP, Magnotti LJ, Weinberg JA, et al. Applicability of an established management algorithm for destructive colon injuries after abbreviated laparotomy: a 17 year experience. J Am Coll Surg 2014;218:636 - 43.

4. Gonzalez RP, Falimirski ME, Holevar MR. Further evaluation of colostomy in penetrating colon injury. Am Surg 2000;66:342- 6 .

5. Cheong JY, A. Keshava. Management of colorectal trauma: a review. ANZ J Surg 2017;87: 547-53. 